\title{
Abgeschliffene Mammutstoßzähne aus dem Emschertal
}

\author{
Von Karl Brandt, Herne \\ Mit 4 Abbildungen im Text
}

$\mathrm{Z}$ u samme $\mathrm{nf}$ a s sung: Es werden zwei Fragmente von Mammut-Stoßzähnen beschrieben, die aus den basalen "Knochen-Schichten" der Emscher-Niederterrasse (letzte Eiszeit) bei Herne und Bottrop entstammen. Beide Stoßzahn-Fragmente zeigen am distalen Ende Schliffmarken, die auf Scharrbewegungen im gefrorenen Schnee oder Sand zurückgeführt werden. Ein drittes Fundstück zeigt beidseitig zur Spitze des Stoßzahn-Fragmentes hin meißelartige Abflachungen. Vermutlich wurde dieser Stoßzahn als Arbeitszahn zum Ausgraben von Pflanzen oder zum Suchen nach Wasser benutzt; vielleicht liegt aber auch ein Artefakt vor. Das vierte Fundstück endlich, allseitig stark gerundet, am proximalen Ende mit poliert erscheinender Oberfläche, stammt aus der Knochenschicht des Schwarzbachtales bei Gelsenkirchen-Hessler. Es wird vermutet, daß es sich hierbei um ein altpaläolithisches Gerät handelt, das wie die bekannten „Glockenschaber" aus Knochen zum Enthaaren der Tierfelle verwandt wurde.

$\mathrm{Su} \mathrm{m}$ m a r y : Two fragments of mammoth tusks, found in a layer rich in bones of the River Emscher in the region of Herne and Gelsenkirchen-Buer, are described. The teeth show marks of scraping, obviously caused either by frozen snow or sand raking, the last being less probable. A third specimen is wedged towards its distal end, and it might have been shaped in this manner as a mammoth used it to dig out platroots or water. A fourth fragment of a tusk, rounded on all sides and with a polished appearance at the proximate end was found at another place. This fragment can not hardly be defined as an artefact of the Neandertal-Period. It probably served for a similar purpose as do the "bell-scrapers" made of bone.

Namentlich durch den Bau des Rhein-Herne-Kanals und die Regulierung der Emscher in den letzten Jahren vor 1914 sind die sogenannten Knochenkiese oder -sande der Emscher bekannt geworden. Damals wurden die Knochen kaltzeitlicher Säuger darin in Menge gefunden. Im Jahre 1959 hatten wir (Emschertalmuseum Herne) Gelegenheit, in den Knochenkiesen eine Ausgrabung zu veranstalten (Neubau der Kläranlage Nord in Herne). Hier haben wir planiert und Schnitte angelegt. Es bestätigte sich, was wir schon lange beobachteten. Die Knochenschicht als unterste Niederterrassenablagerung der Emscher liegt nicht dem bankigen Emschermergel auf, sondern wird von diesem durch eine $20-40 \mathrm{~cm}$ mächtige graue, tonige Schicht aufgeweichtem Emschermergel getrennt. In diese Tonschicht sind bisweilen einzelne Knochen, Geschiebe und Gerölle hineingeraten. Darauf liegt die eigentliche Knochenschicht, die $10--50 \mathrm{~cm}$ mächtig sein kann. Gerölle liegen darin in Schmitzen, nie in durchgehender Lagerung. Abgedeckt wird die Knochenschicht durch dunkelockerfarbige, eisenschüssige Sande, die selten kleinere Knochen enthalten (Schnekkensande). In der unterlagernden tonigen Schicht wurden bisweilen flache Wannen beobachtet, in denen sich Knochen gehäuft angesammelt hatten; diese wurden eingeschwemmt, konnten jedoch nur bei stärkerem Wasserdruck daraus wieder ausgeschwemmt werden.

In einer solchen Wanne fanden wir in der genannten Kläranlage auch das maximal $1 \mathrm{~m}$ lange distale Ende eines Mammutstoßzahnes (vgl. Abb. 1), das alt abgebrochen ist (Original im Emschertalmuseum Herne). Der Umfang des Bruchstückes beträgt am proximalen Ende $36 \mathrm{~cm}$. Von der Bruchstelle bis zur Zahnspitze in der Waagerechten gemessen, ist das Bruchstück nur $75 \mathrm{~cm}$ lang. Von dieser Waagerechten in der Mitte $(37,5 \mathrm{~cm})$ senkrecht nach unten auf die Innenseite der Stoßzahnbiegung gemessen, ist unter Fund $19 \mathrm{~cm}$ gebogen, bis zur Unterkante gemessen $29 \mathrm{~cm}$.

An diesem Stoßzahnbruchstück fällt sofort auf, daß die Spitzenpartie auf eine Länge von $45 \mathrm{~cm}$ wie flachgedrückt erscheint. Zur Spitze hin wird sie immer flacher. Eine nähere Betrachtung dieser Erscheinung ergibt folgendes: Höchstwahrscheinlich handelt es sich um den linken Stoßzahn, dessen aus den Alveole herausragende Länge auf etwa $2 \mathrm{~m}$ geschätzt werden kann. Die untere Seite, namentlich links außen, ist auf eine Länge von $32 \mathrm{~cm}$ ab- 


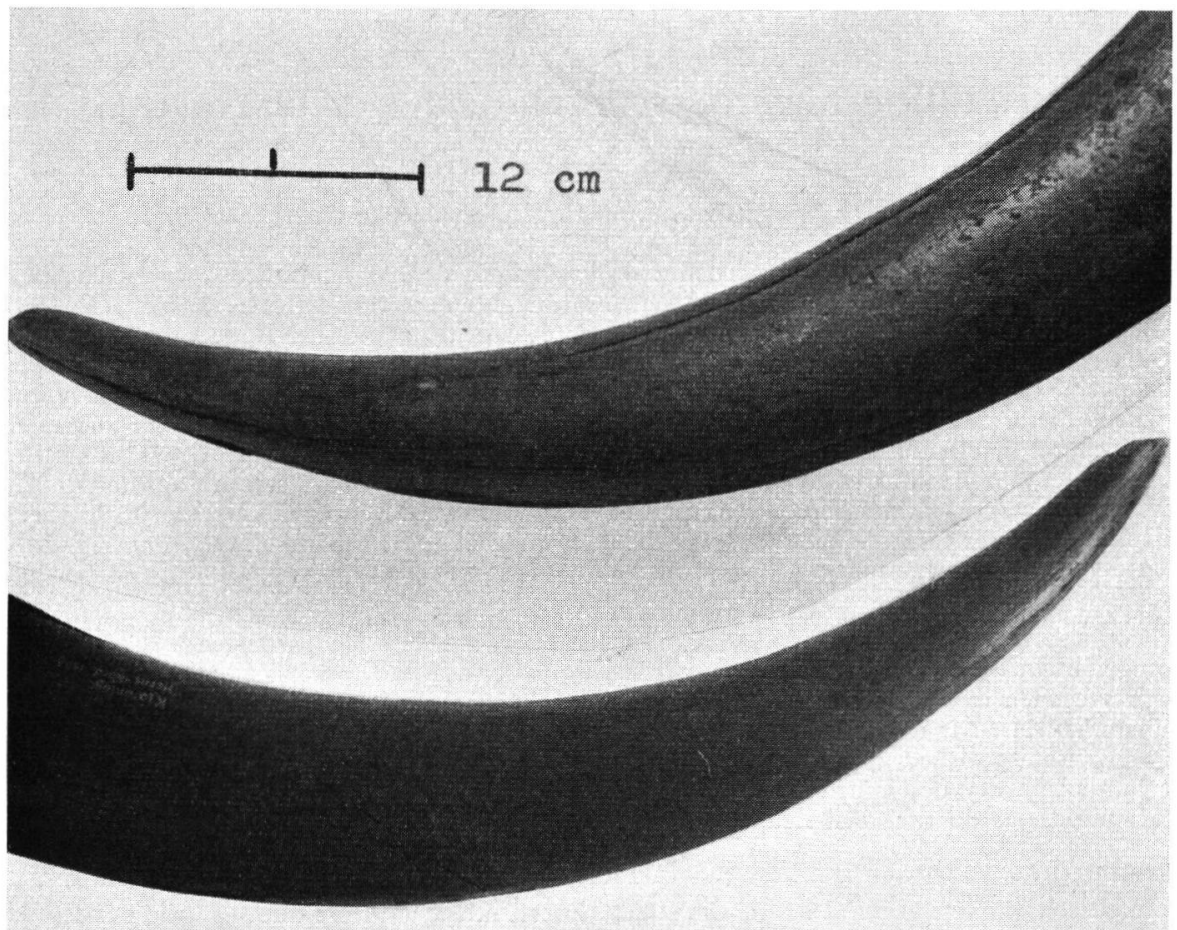

Abb. 1. Abgeschliffene Stoßzahnspitze aus den Knochenkiesen der Emscher von Herne-Nord. Oben: die linke, am meisten abgeschliffene Seite. Unten: dasselbe Stoßzahn-Fragment von der rechten Seite gesehen. Foto: K. BRandT.

geflacht, und zwar beträgt die Abflachung bis zur Zahnspitze $1 \frac{1}{2}$ bis $2 \mathrm{~cm}$. Aber auch die Oberseite des Zahnes hat eine, wenn auch geringe Abflachung erfahren, die nach links außen etwas zunimmt.

Diese Abflachung des distalen Stoßzahnendes ist zweifelfrei durch allmähliches Abschleifen entstanden. Dafür zeugen noch zwei weitere Befunde. Erstens ist diese Zahnpartie ringsum geglättet, was ohne weiteres auffällt, und zweitens befinden sich auf der geglätteten Unterseite im Bereich der genannten $32 \mathrm{~cm}$, quer zur Zahnlänge, zahlreiche bis $4 \mathrm{~cm}$ lange Schrammen, die auf der ebenfalls geglätteten Oberseite nicht vorkommen! Dieser Fall ist klar: das Mammut hat in den Wintern der Kaltzeit mit den Stoßzähnen den Schnee fortgescharrt, um an die dürftige Pflanzennahrung zu gelangen. Das Tier hat offensichtlich von rechts nach links gescharrt, wodurch die weitaus intensivere Abschleifung an der linken Außenpartie der Stoßzahnspitze erfolgte. Diese bemerkenswerte Abschleifung kann unmöglich in einem Winter erfolgt sein, denn dafür ist die äußere Schale eines Stoßzahnes zu hart und die Abschleifung zu intensiv. Es kann angenommen werden, daß die Schneedecke wenigstens zeitweise gefroren war, wodurch wahrscheinlich die Abschleifung begünstigt wurde. Aber auch ein Scharren in Sandböden kann in Erwägung gezogen werden.

Noch ein zweiter Mammutstoßzahn mit den gleichen, aber viel stärker ausgeprägten Erscheinungen liegt aus dem Emschertal vor (vgl. Abb. 2). Um den Rhein-Herne-Kanal von Zuschwemmungen zu säubern, wurde bei Bottrop ausgebaggert. Die hierbei zum Vorschein gekommenen, vielen Knochen pleistozäner Säuger hat Arno HEINRICH, Leiter des Heimatmuseums Bottrop, dankenswerterweise geborgen. All diese Funde stammen aus der Knochenschicht der pleistozänen Emscher. Unter den Bottroper Fundsachen befindet 


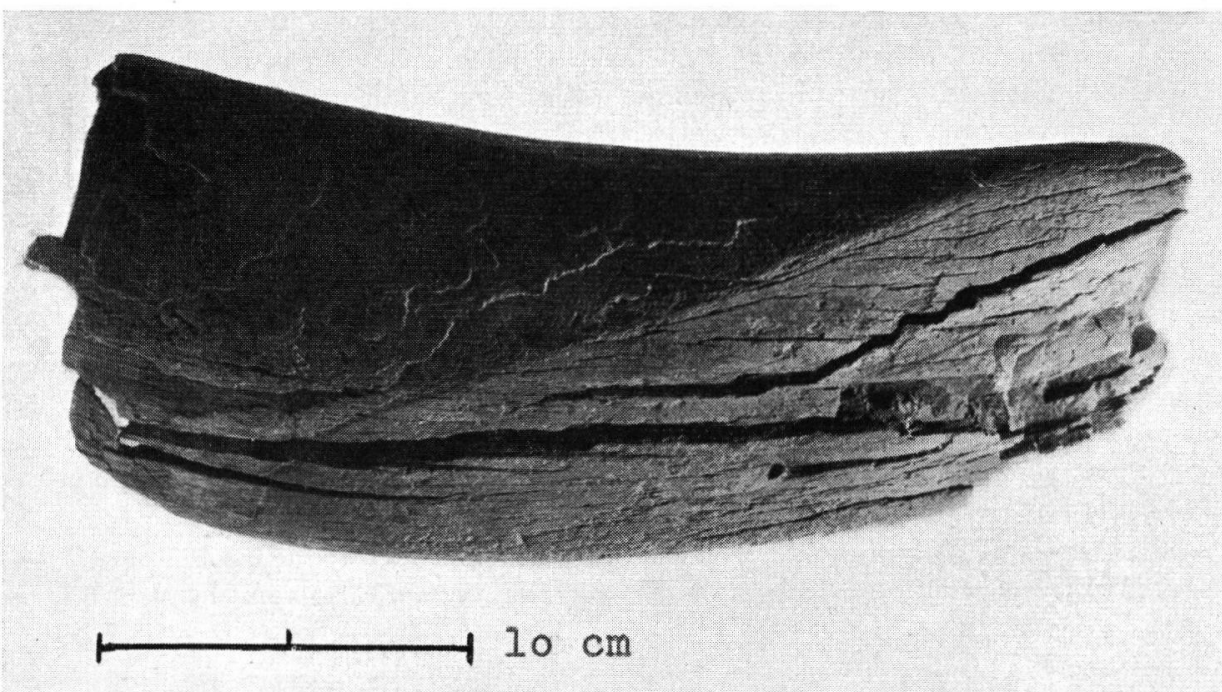

Abb. 2. Abgeschliffene Stoßzahnspitze aus den Knochenkiesen der Emscher von Bottrop-Süd (RheinHerne-Kanal). Die Abschliff-Fläche ist kürzer, aber viel stärker ausgebildet als auf dem in Abb. 1 dargestellten Fundstück; auch hier ist wieder besonders die linke Partie der Schliff-Fläche (Bildaufsicht) stärker ausgeprägt. Links im Bild: rezente Bruchfläche.

Foto: K. BRANDT.

sich das frisch (durch Bagger) abgebrochene, jetzt maximal $34,5 \mathrm{~cm}$ lange distale Ende eines starken Mammutstoßzahnes. Am proximalen Ende besitzt es einen Umfang von $38,5 \mathrm{~cm}$. Da von der Spitze des Zahnes einige Zentimeter fehlen (vermutlich $3-4 \mathrm{~cm}$ ), beträgt jetzt die Länge der abgeschliffenen Fläche $30 \mathrm{~cm}$.

Bei diesem Fundstück handelt es sich um einen rechten Stoßzahn. Auch der Träger dieses Stoßzahnes hat offensichtlich die Scharrbewegungen von rechts nach links ausgeführt, da die stärkste Abschleifung an der Unterseite links liegt.

Während der zuerst beschriebene abgeschliffene Stoßzahn von einem weiblichen Tier stammen dürfte, haben wir es bei dem Bottroper Fundstück wohl mit einem männlichen Tier zu tun, worauf die Stärke des Stoßzahnes hinweist. Eine eigentliche Spitze ist daran nicht mehr vorhanden, weil sie abgeschliffen worden ist. Dadurch fehlt von der ursprünglichen Länge ein größeres Stück. Dieser Stoßzahn dürfte trotz seiner Stärke kürzer gewesen sein als der viel schlankere von Herne, wodurch die Spitzenpartie stärker der Abschleifung unterworfen worden ist. Der Grad der Abschleifung wird ersichtlich, wenn der ursprüngliche Umfang am distalen Ende angegeben wird. Wir errechnen ungefähr $30 \mathrm{~cm}$. Davon sind in der Senkrechten etwa maximal $4-5 \mathrm{~cm}$ abgeschliffen worden. Die Breite der Schlifffläche beträgt im Bereich des Spitzenendes $10 \mathrm{~cm}$. Querschrammen sind auf der Schlifffläche nicht festzustellen. Dieses interessante einmalige Fundstück befindet sich im Heimatmuseum von Bottrop.

$\mathrm{Zu}$ diesen beiden beschriebenen abgeschliffenen Stoßzähnen muß ausdrücklich bemerkt werden, daß es sich um Schliffspuren und nicht um Grab- oder Stoßspuren handelt, wie letztere bisweilen an Arbeitszähnen von rezenten Elefanten vorkommen, zumal die Schliffspuren eindeutig quer zur Länge der Mammutstoßzähne verlaufen. Bei der Verwendung zu Grab- und Stoßarbeiten hätten (wie bei unserem Fundstück Nr. 3) Abnutzungsspuren frontal vom distalen Ende in Längsrichtung zum proximalen Ende der Incisoren vorhanden sein müssen. Beim Ausgraben z. B. von Krähenbeeren- und Ericaceenbüschel, sowie

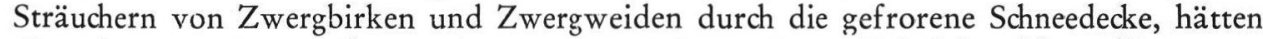
die zuletzt genannten Abnutzungsspuren entstehen müssen. Dabei ist nicht völlig auszu- 
schließen, daß sie gelegentlich auch dazu gedient haben (briefliche Mitteilung von E. GüNTHER), wie kleine alte Absplitterungen an der Endspitze des zuerst beschriebenen Zahnes ausweisen könnten, falls sie zu Lebzeiten des Trägers entstanden sind ${ }^{1}$ ).

Im Heimatmuseum Bottrop befindet sich ferner das $19 \mathrm{~cm}$ lange distale Bruchstück eines kleinen Stoßzahnes (vgl. Abb. 3), das am proximalen Ende einen Umfang von $15 \mathrm{~cm}$ hat. Offensichtlich handelt es sich um den Rest eines rechten Stoßzahnes eines jüngeren T'ieres. Auf eine Länge von etwa $7 \mathrm{~cm}$ ist die Spitze beidseitig (rechts und links in Normallage) zum proximalen Ende hin abgeschrägt, wodurch das Fundstück wie ein Meißel erscheint (Abb. 3). Die beiden angeschrägten Seitenflächen sind offenbar nicht abgeschliffen, sondern abgestoßen worden, zumal beide Schrägflächen breite, tiefe Schrammen auf weisen, die an der linken Seite (Außenseite) besonders breit (bis $4 \mathrm{~mm}$ ) und tief sind (1 mm). Diese Breitschrammen verlaufen nicht parallel mit den Abschrägungen, sondern liegen schräg zu derselben, von der Oberkante des Stoßzahnes an der Spitze zur Unterkante dem proximalen Ende zu. Dies ist an der linken Schrägung der Fall (Abb. 3, 2 Pfeile unten).

Anders verlaufen die dichter liegenden Schrammen an der rechten Schrägung. Diese verlaufen entgegengesetzt zu denen auf der linken Schrägung, also vom distalen weniger

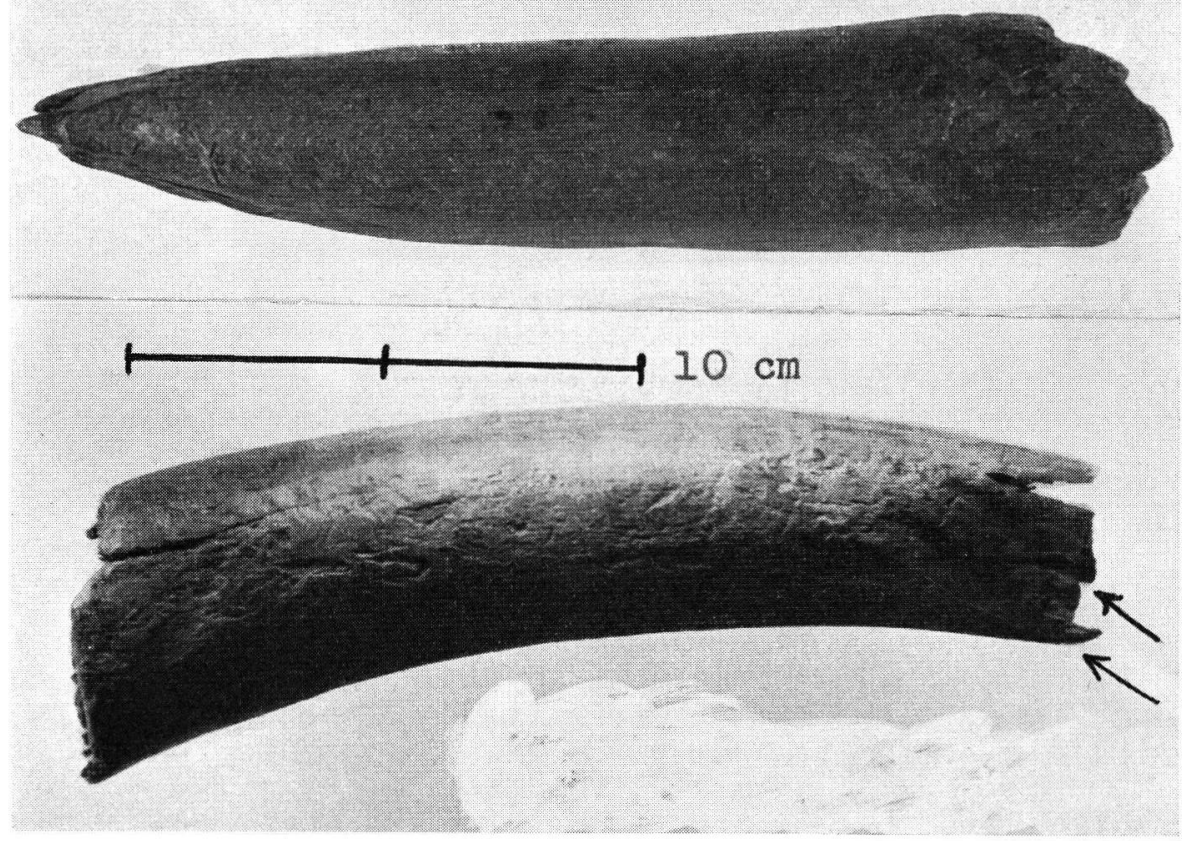

Abb. 3. Abgeschliffene Stoßzahnspitze aus den Knochenkiesen der Emscher von Bottrop-Süd (RheinHerne-Kanal). Sie wird als Arbeitszahn eines Mammuts angesehen. Oben: das Fundstück in der Aufsicht, die meißelartige Ausbildung der Spitze ist deutlich sichtbar. Unten: dasselbe Fundstück von der Seite gesehen; beide Pfeile weisen auf schräg nach oben verlaufende Rillen hin, die beim Graben im Erdreich entstanden sein dürften.

Foto: K. Brandt.

1) Das in Abb. 1 dargestellte Fundstück hat E. Guenther, Kiel, Fl. Heller, Nürnberg, und L. Zotz, Erlangen, vorgelegen, die die Schliffspuren an dem Stoßzahnfragment als Scharrspuren anerkannten. P. Siegried, Münster, teilte mit, daß O. ABEL und K. BEurLen die Benutzung von Mammutstoßzähnen als Werkzeuge zum Schneescharren zumindest von Tieren im höheren Alter verneint hätten, ebenso auch H. F. OsBonN; dagegen wären jedoch einige russische Forscher der Ansicht, daß das Mammut seine Incisoren zum Schneescharren verwandte, so W. GARUTt und W. Dubinin im Zool. Journ. 30, Leningrad 1951, wo allerdings nur mit wenigen Sätzen auf derartige "Abnutzungszonen" an Mammutstoßzähnen hingewiesen wird. 
schräg zum proximalen Ende. Man könnte versucht sein, dieses Zahnfragment als ein Artefakt eines pleistozänen Menschen anzusehen. Aufgrund der Zugehörigkeit der Knochenschicht zum Anfang der letzten Kaltzeit (Würm) käme ein Neandertaler in Frage. Betrachten wir das proximale Ende genauer, so scheint es, als ob ein 3,5 cm langer Einschnitt in die äußere Stoßzahnschale vorhanden wäre (Abb. 3, linke Karte).

Wir möchten die Frage offenlassen, ob ein Artefakt vorliegt; vielleicht war es ein Arbeitszahn, wie sie von unseren heute noch lebenden Elefanten bekannt sind, die damit Rinde von den Bäumen stoßen oder diese gar fällen. Eine allseitige Glättung an diesem zweiten Bottroper Fundstüück ist nicht zu übersehen, aber Bearbeitungsspuren, um die meißelartige Schneide im Spitzenbereich herzustellen, sind nicht mit Sicherheit zu erkennen.

Bei dem vierten hier zu beschreibenden Stoßzahnfragment handelt es sich vielleicht um ein Artefakt. Es ist wieder das distale Ende eines Mammutstoßzahnes von jetzt $43 \mathrm{~cm}$ Länge. Das Fundstück ist in alter Zeit der Länge nach aufgespalten, so daß die Zuwachskegel im Schnitt freiliegen. Gefunden wurde es vom Verfasser in der Knochenschicht des Schwarzbaches in Gelsenkirchen-Heßler, kurz vor der Einmündung in die Emscher (bei der Regulierung des Schwarzbaches 1953). Das Fundstück befindet sich im Emschertalmuseum in Herne. Bemerkenswert daran ist das allseitig völlig abgerundete proximale Ende dieses Stoßzahnfragmentes. Die Abrundung ist durch Abschliff erfolgt, aber auf keinen Fall durch Abrollung in der Knochenschicht. Dagegen spricht, daß diese Abschleifung nicht in die Höhlung des proximalen Endes hineinreicht. Dieses Ende kann mehr oder weniger senkrecht auf irgendeinen zu bearbeitenden Werkstoff aufgesetzt worden und dann auf diesem hin- und hergezogen worden sein, vielleicht mit kreisenden Bewegungen. Hierbei konnte wohl der Rand in der Aufsicht und nach außen abgeschliffen, also gerundet werden, nicht aber nach innen. Diese Abschleifung ist an der Außenwandung nicht gleichmäßig; stellenweise reicht sie weit hinunter. Im Bereich der Rundung des Randes erscheint dieser Teil wie glänzend poliert.

Der polierte Rand des proximalen Endes liegt ringsum nicht waagerecht, sondern verläuft von der äußeren Krümmung zur inneren schräg. Diese Abschrägung macht $2 \mathrm{~cm}$ aus. Wenn wir uns nun dieses Fundstück als in der Art der bekannten „Glockenschaber" aus Knochen verwendet vorstellen, die zum Enthaaren und Glätten der Felle verwendet wor-

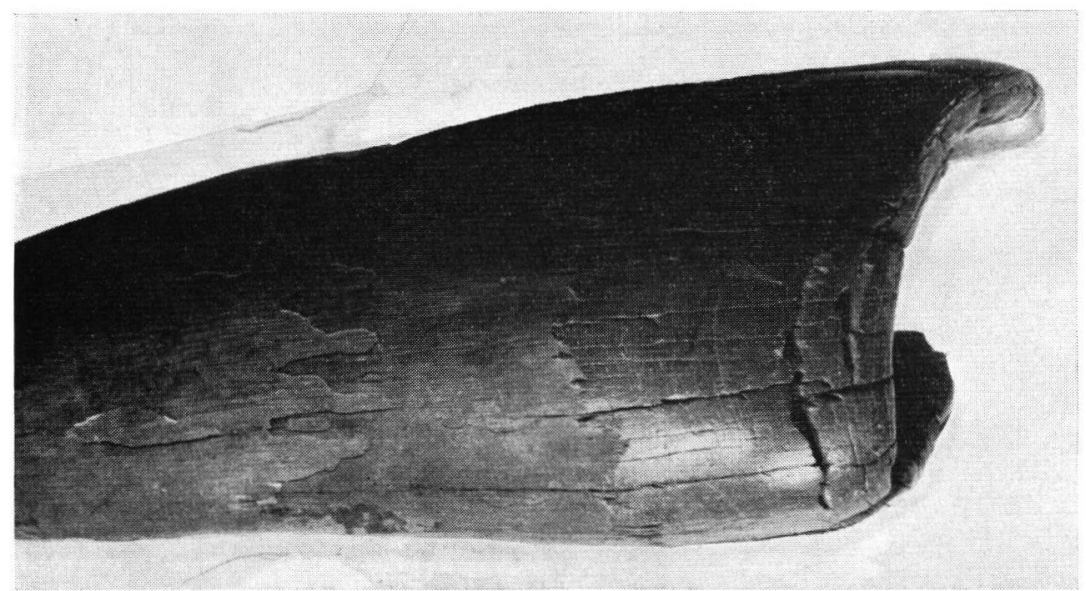

Abb. 4. Abgeschliffenes proximales Ende eines Stoßzahn-Fragmentes aus den Knochenkiesen des Schwarzbachtales von Gelsenkirchen-Heßler. Dieses Stoßzahn-Fragment ist vielleicht als „Glockenschaber"- Artefakt zum Enthaaren von Fellen anzusprechen. Die durch Abschliff entstandene Rundung am Bruch des proximalen Endes (oben) ist deutlich zu erkennen und erscheint wie auf Hochglanz poliert. Der hintere, fehlende Stoßzahnteil ist rezent ausgebrochen. $x \frac{1}{2}$. Foto: K. BRAndt. 
den sein dürften, so war diese Abschrägung nicht unvorteilhaft für den Nutzungseffekt, zumal es darauf ankam, daß der Rand voll auf dem Werkmaterial auflag. Da nun, wie erwähnt, die Abschrägung zur inneren Krümmung verläuft, war bei dem Arbeitsvorgang die Spitze des Stoßzahnes zum damit arbeitenden Menschen gewandt, was die Arbeit erleichterte; denn wäre der Rand vollständig waagerecht, hätte das Fundstück senkrecht aufgestellt werden müssen, womit die Arbeit erschwert worden wäre. Das Stück kann auf dem Werkstück schräg zum arbeitenden Menschen gestanden haben, denn jener zum Menschen liegende Teil des Randes ist weit stärker abgeschliffen, als die übrigen Teile.

Nun ist dieses Stück für einen "Glockenschaber" der herkömmlichen Art viel zu lang. Daher wird vermutet, daß das dem Arbeitsende gegenüberliegende Ende gegen das rechte Schlüsselbein des mutmaßlich damit arbeitenden Menschen gelehnt wurde, was vielleicht den Arbeitseffekt erhöhte.

Hierzu hat auch noch folgender Umstand beigetragen. In der genannten Stellung wurde unser Stück geschoben, also vom Körper des Menschen fort und wieder herangezogen. Die Zugkraft wirkte sich wie bei allen Artefakten weit mehr aus als die Schubkraft, abgesehen, daß beim Ziehen hauptsächlich der vordere Randteil sich im Nutzungseffekt auswirkte, weil sich der hintere Randteil rein mechanisch etwas anhob. Dadurch wurde der vordere Randteil weit mehr abgeschliffen, weil ein größerer Teil von ihm auflag. Die Ansprache dieses Fundes als Artefakt ist natürlich problematisch, aber zur Zeit ist keine andere Deutung zu finden ${ }^{2}$ ).

\section{Schriftenverzeichnis}

AndreE, J.: Das Alter der diluvialen Lippe- und Emscherablagerungen. - - Sber. naturhist. Ver. Rheinl. Westf., S. 14-28, Bonn 1927.

BärtLING, R.: Das Diluvium des niederrheinisch-westfälischen Industriebezirks und seine Beziehungen zum Glazialdiluvium. - Z. deutsch. geol. Ges. 64, Mon.-Ber. 3, S. 155-177, Berlin 1913.

BRANDT, K.: Über die Vorkommen von Säugerknochen in pleistozänen Ablagerungen des Ruhrgebietes. - Natur u. Heimat 13, S. 78-81, Münster 1953.

KAHrs, E.: Zur Kenntnis des Emscherdiluviums. - Die Heimat (Z. westf. Heimatbundes), A. 7, S. $211-213,1928$.

Kukuk, P.: Über den Fund eines Schädels vom Moschusochsen im Diluvium des Emschertales. Z. deutsch. geol. Ges. 65, S. 597-600, Berlin 1914.

Menzel, H.: Die Quartärfauna des niederrheinisch-westfälischen Industriebezirkes. - Z. deutsch. geol. Ges. 64, Mon.-Ber. 3, S. 177-200, Berlin 1913.

Manuskr. eingeg. 8. 4. 1962.

Anschrift des Autors: Karl Brandt, Herne i. Westf., Emschertalmuseum im Schloß Strünkede.

2) Dem Verfasser sind vor ca. 30 Jahren aus dem Emschertal noch zwei ganz gleiche „StoßzahnArtefakte" bekannt geworden, nämlich im Niederrheinischen Museum in Duisburg-Hamborn und im Heimathaus Gelsenkirchen. 